\title{
Aggregation of SOA and Cloud Computing: A Recent Emerging Technology
}

\author{
Raghvendra Kumar ${ }^{1}$, Prasant Kumar Pattnaik ${ }^{2}$, Priyanka Pandey ${ }^{3}$ \\ ${ }^{1}$ Dept. of Computer Science and Engineering, Lakshmi Narain College of Technology, Jabalpur, India \\ ${ }^{2}$ School of Computer Engineering, Kalinga Institute of Industrial Technology University, Bhubaneswar, Odisha, India \\ ${ }^{3}$ Dept. of Computer Science and Engineering, Takshshila Institute of Engineering and Technology, Jabalpur, India
}

\section{Email address:}

Raghvendraagrawal7@gmail.com (R. Kumar),patnaikprasantfcs@kiit.ac.in (P. K. Pattnaik), pandeypriyanka906@gmail.com (P. Pandey)

\section{To cite this article:}

Raghvendra Kumar, Prasant Kumar Pattnaik, Priyanka Pandey. Aggregation of SOA and Cloud Computing: A Recent Emerging Technology. International Journal on Data Science and Technology. Vol. 2, No. 1, 2016, pp. 5-8. doi: 10.11648/j.ijdst.20160201.12

\begin{abstract}
Cloud is gaining popularity as means for saving cost of IT ownership and accelerating time to market due to ready-to-use, dynamically scalable computing infrastructure and software services offered on Cloud on pay-per-use basis. Design of software solution for delivery as a shared service over Cloud requires specific considerations. Cloud computing is a way to increase the capacity or add capabilities dynamically without investing in new infrastructure, training new personnel, or licensing new software. It extends Information Technology's (IT) existing capabilities. In the last few years, cloud computing has grown from being a promising business concept to one of the fast growing segments of the IT industry. But as more and more information on individuals and companies are placed in the cloud, concerns are beginning to grow about just how safe an environment it is. Despite of all the hype surrounding the cloud, enterprise customers are still reluctant to deploy their business in the cloud. Security is one of the major issues which reduces the growth of cloud computing and complications with data privacy and data protection continue to plague the market. The advent of an advanced model should not negotiate with the required functionalities and capabilities present in the current model. A new model targeting at improving features of an existing model must not risk or threaten other important features of the current model. The architecture of cloud poses such a threat to the security of the existing technologies when deployed in a cloud environment. Cloud service users need to be vigilant in understanding the risks of data breaches in this new environment. In this paper, a survey of the different exiting solution and that pose a new solution to the cloud is presented. In this paper we describe an approach for design of infrastructure resource management as a service for use by group of institution based on Service Oriented Architecture, Software-as-a-Service, and Cloud Computing paradigms.
\end{abstract}

Keywords: Cloud Computing, SOA, Role of SOA in Cloud Computing, Aggregation of SOA and Cloud Computing

\section{Introduction}

Service Oriented Architecture and Cloud Computing are two popular design paradigms in order to fulfill the user's requirement that includes for high cost and heavy infrastructure applications. SOA is demanding for the different in built approach and cloud is includes for security and infrastructure measures. In this paper, we try to focus the functionality of two widely used approaches. Again we aim to aggregate this two approaches for low cost and efficient access of infrastructures [1].

Service Oriented Architecture is an architectural design and discipline that may be used to build infrastructures enabling those with requires and those with capabilities to interact via services across disparate domains of technology and ownership. Services act as the core facilitator of electronic data interchanges yet require additional mechanisms in order to function. Several new trends in the computer industry rely upon SOA as the enabling foundation. These include the automation of Business Process Management (BPM), composite applications The latter, Web is not defined as a static architecture. Web can be generally characterized as a common set of architecture and design patterns, which can be implemented in multiple contexts. [1] The list of common patterns includes the Mashup, Collaboration-Participation, and Software as a Service (SaaS), Service Oriented Architecture (SOA) is a paradigm for organizing and utilizing distributed capabilities that may be under the control of different ownership domains and implemented using various technology stacks. In general, 
entities (people and organizations) create capabilities to solve or support a solution for the problems they face in the course of their business [3]. It is natural to think of one person's needs being met by capabilities offered by someone else; or, in the world of distributed computing, one computer agent's requirements being met by a computer agent belonging to a different owner. The term owner here may be used to denote different divisions of one business or perhaps unrelated entities in different countries [5].

\section{Service Oriented Architecture}

Reference architecture is a more concrete artifact used by architects. Unlike the reference model, it can introduce additional details and concepts to provide a more complete picture for those who may implement a particular class. Reference architectures declare details that would be in all instances of a certain class, much like an abstract constructor class in programming. Each subsequent architecture designed from the reference architecture would be specialized for a specific set of requirements. Reference architectures often introduce concepts such as cardinality, structure, infrastructure, and other types of binary relationship details. Accordingly, reference models do not have service providers and consumers [5]. If they did, then a reference model would have infrastructure (between the two concrete entities) and it would no longer be a model.

Software architecture has been emerging as a discipline over the last decade. A system's software architecture describes its coarse-grained structuresand its properties at a high level. As long as the technology supports thosestructures and properties, the technology can be considered to implement the architecture. For instance, Jini is a technology that supports service-oriented architecture, because it supports the properties of SOA. It is important to apply the concepts of software architecture to any new technologyto take full advantage of it. Service-oriented architecture is implementedby technologies other thanWeb services, but the term and concepts have gained popularity recently because of Web services. Interface design is perhaps the most difficult part of designing services in service-oriented architecture [6]. The modularization techniques practiced for decadesstill apply to services. Service design is even more difficult, because the domain aservice supports is not limited to a single application. Therefore, it is best to performmodularization starting with a conceptual model of the business ratherthan of a single application. If the interface design is done well, the services aremore likely to be reusable in other applications, and organizations will realize ahigher return on their investment.Web services are refocusing organizations on the concepts of service-orientedarchitecture. Although highly reusable, loosely coupled architectures have beena goal for many organizations. Web services are fostering interest in and providingthe technology to implement service-oriented architectures that enable themto realize their vision.

\section{SOA Application}

Service-oriented architecture (SOA) describes a category of composite applications composed of service provider and service consumer components. SOA segregates business logic and offers location transparency for the service providers and consumers [7].

The SOA approach lets you replace or upgrade individual components in the application without affecting other components or the process as a whole. Moreover, you can independently specify alternative paths through which the components in the application exchange messages.

- To manage a world of data: An SOA-focused deployment is helping the National Aeronautics and Space Administration to streamline access to its mounds of data, as well as link users of the data to the services they need to process the data.

- To manage workflows: A BPEL-based workflow system was employed at State Children's Health Insurance Program to manage the nine-step application process that determines and responds to applicants' eligibility.

- To consolidate services: Harvard Medical School and its hospital affiliates radically streamlined their business processes around the sharing of medical data by building an SOA involving about 25 categories of Web services shared between 400 different departments with 14,000 employees. Seattle's 17-hospital Providence Health System is leveraging Web services to link its inhouse legacy systems into a single patient portal, permitting online bill paying among other services.

- To improve customer service: Starwood Hotels and Resorts Worldwide is replacing its legacy roomreservation system with an SOA-based one, going live with as many as 150 service-based applications built on Web standards.

- For more effective partnerships: T-Mobile is employing SOA for both internal integration and reuse, as well as the external, partner- and revenue-generating elements. This approach enables T-Mobile to work effectively with third-party content providers such as Time Warner and the Bertelsmann Group to deliver services to customers.

- To trim costs: Verizon Communications claims it averages about 2.5 million to three million Web services transactions a day through a "home-grown" SOA. The system went operational in 2004 and Verizon says it has slashed its IT budget by $50 \%$ by eliminating redundant systems inherited from the merger of Bell Atlantic and GTE. The SOA also helped integrate the operations of some 7,000 developers.

- To increase speed to market: Owens \& Minor, a distributor of medical and surgical supplies, has embarked on a four-year SOA initiative, and forecasts annual savings of $\$ 650,000$, half attributable to better inventory accuracy and half to productivity savings and improved cash flow. Leveraging SOA and BPM, the company can now automate processes in a few weeks 
that once would have taken as much as nine months.

- For strategic differentiation: Fireman's Fund is relying on SOA to consolidate $70 \%$ of its technology applications. By more effectively aligning business with IT and strengthening its relationship with agents, Fireman's Fund believes it can strategically differentiate itself in a crowded property and casualty marketplace.

- To increase agility: Motorola has introduced 180 services through its SOA framework and business activity monitoring projects (monitoring the linkages between enterprise software apps), and has an average of 50 rules, covering everything from credit card transactions to warranty services.

- To loosely couple businesses: Mohan Sawhney, professor at Northwestern's Kellogg School of Management, says the best-run companies may not be producers themselves, but networks of producers, orchestrated by a front-end broker of services. Some mobile phone companies already "don't do anything themselves, they just collect the money.

\section{Role of SOA in Cloud Computing}

In its broadest usage, the term cloud computing refers to the delivery of scalable IT resources over the Internet, as opposed to hosting and operating those resources locally, such as on a college or university network. Those resources can include applications and services, as well as the infrastructure on which they operate. By deploying IT infrastructure and services over the network, an organization can purchase these resources on an as-needed basis and avoid the capital costs of software and hardware. With cloud computing, IT capacity can be adjusted quickly and easily to accommodate changes in demand. While remotely hosted, managed services have long been a part of the IT landscape, a heightened interest in cloud computing is being fueled by ubiquitous networks, maturing standards, the rise of hardware and software virtualization, and the push to make IT costs variable and transparent [8].

Cloud and cloud-like solutions appear to be widespread and growing in higher education, though in relatively focused areas, such as student e-mail. E-mail notwithstanding, higher education institutions are more likely to obtain new services from the cloud than to transition established services that have long been operated by the campus. Many colleges and universities see pockets of cloud service usage in other areas, often led by individual faculty or students looking for the added flexibility and convenience that the cloud can provide. Among the drivers that are encouraging more institutions to contemplate cloud services are budget pressures, calls for increased reliability of and access to IT systems, and the need for institutions to provide timely access to the latest IT functionality [10].

In traditional enterprise computing, IT departments forecast demand for applications and capacity and invest time and money to develop those resources in-house or purchase them from others and operate them in-house. With cloud computing, institutions procure IT services from remote providers, and campus constituents access these resources over the Internet. E-mail, for example, long considered a staple of an institution's IT operations, can be obtained from a range of sources, and a growing number of campuses contract with outside suppliers for this function. Software is hosted by the provider and does not need to be installed-or maintained - on individual computers around campus [2]. In some cases, a large university or a consortium might become a provider of cloud services. Storage and processing needs can also be met by the cloud. Institutions pay only for the resources used, and users can access the applications and files they need from virtually any Internet-connected computer. In a mature cloud computing environment, institutions would be able to add new IT services or respond to changes in capacity on the fly, saving capital costs that can be redirected to programs of strategic value to the institution.

Agregation of SoA and Cloud Computing:

Software design should be virtually implemented, parallel, and reliable. They must be capable of distributing workload. This project must be designed for customizing and reducing effort and cost in order to mark up with business flow. It also requires certain rules, processing logic, user interface, security, reliability and back up or fault tolerance. This project must be handling within a specific range.

A. Problem Area.

Cloud computing introduces significant concerns about privacy, security, data integrity, intellectual property management, audit trails, and other issues. Because higher education is subject not only to institutional policies but also to a broad range of state and federal regulations, these issues are complex and become even more difficult in the context of inter-institutional cloud initiatives. Because of the control that consumers of cloud services cede to providers, successful initiatives rely on a high degree of trust between a college or university and a supplier, including confidence in the provider's long-term viability [8].

\section{Conclusion}

In this paper, several cloud Computing paradigms have been in-sighted. Cloud Computing and SOA is an evolution of thesetechnologies and has heralded not to replace any existingtechnology. Each technology has its own place and differenttechnologies need to blend together in the IT environments. Clouds intend to provide additive features and solutions tothe problems that haunt these technologies. Several obstaclesin the way of Cloud Computing have been identified in thiswork and future directions have been proposed so thatadvantages of the Cloud can be unleashed.

\section{References}

[1] A study on $3 \mathrm{G}$ and $4 \mathrm{G}$ technology by Shrabanee Swagatika, Dr. Prasanta Kumar Pattnaik, Ramesh Chandra Sahoo, A journal of decision making, siddhant, 2010, pg no. 71-73. 
[2] Design issues of simple cloud based software as a service using $3 \mathrm{G}$ and $4 \mathrm{G}$ technology by Shrabanee Swagatika, Dr. Prasanta Kumar Pattnaik, international conference on Electronic Transport, ICET-2011, Pg no. 10-14.

[3] Software as a service, Wikipedia.

[4] Welcome to the Data Cloud, Semantic Web blog, ZDnet, 6 Oct 2008.

[5] Any any any old data, Paul Walk's blog, 7 Oct 2008.

[6] Hand, Eric. "Head in the Clouds." Nature. 25; 449 (2007 Oct).

[7] Pollette, Chris. "How the Google-Apple Cloud Computer Will Work." Howstuffworks.com. 2 Mar. 2008.

[8] Rubel, Steve. "The MacBook Air is the Biggest Test Yet for Cloud Computing." MicroPersuasion. 2 Mar. 2008.

[9] Venezia, Paul. "Product review: MacBook Air is light as, well, air." InfoWorld.com (Feb 11, 2008): NA. General One File. Gale. University of Texas at Austin. 2 Mar. 2008.

[10] Weiss, Aaron. Computing in the clouds. Net Worker 11, 4 (Dec. 2007), 16-25.

[11] S. Hashimi, 2003. Service-oriented Architecture explained.
[12] E. Newcomer and G. Lomow, 2004. Understanding SOA with Web Services.

[13] M. Papazoglou, 2003. Service-oriented computing: Concepts, Characteristics, and Directions.

[14] M. Papazoglou and D. Georgakopolous, 2003. ServiceOriented Computing, Communication of the ACM, Vol. 46, No. 10.

[15] C. Ghezzi, M. Jazayeri, D. Mandrioli, 2003. Fundamentals of software engineering, Prentice Hall.

[16] Craig Balding, "ITG2008 World Cloud Computing Summit", 2008.

[17] Alistair Croll, "Why Cloud Computing Needs Security", 2008.

[18] Jonothan Erickson, "Best Practices for Protecting Data in the Cloud", 2008.

[19] Jon Brodkin, "Seven Cloud-Computing Security Risks", 2008.

[20] Elinor Mills, "Cloud Computing Security Forecast: Clear Skies", 2009.

[21] Geva Perry, "How Cloud \& Utility Computing Are Different", 2008. 